\title{
Mitochondrial carriers and pores: Key regulators of the mitochondrial apoptotic program?
}

\author{
Michal Schwarz • Miguel A. Andrade-Navarro • \\ Atan Gross
}

Published online: 15 February 2007

(C) Springer Science + Business Media, LLC 2007

\begin{abstract}
Mitochondria play a pivotal role in the process of apoptosis. Alterations in mitochondrial structure and function during apoptosis are regulated by proteins of the BCL-2 family, however their exact mechanism of action is largely unknown. Mitochondrial carriers and pores play an essential role in maintaining the normal function of mitochondria, and BCL-2 family members were shown to interact with several mitochondrial carriers/pores and to affect their function. This review focuses on the involvement of several of these mitochondrial carriers/pores in the regulation of the mitochondrial death pathway.
\end{abstract}

Keywords Apoptosis - Mitochondrial carrier proteins . Mitochondrial pores $\cdot$ BCL-2 family members · BID . Mitochondrial carrier homolog $2($ Mtch2) · ADP/ATP translocase (ANT) $\cdot$ Voltage dependent anion channel (VDAC)

\section{Apoptosis: an overview}

Apoptosis is essential for normal development and maintenance of tissues. Insufficient apoptosis can lead to cancer

\footnotetext{
M. Schwarz · A. Gross $(\triangle)$

Department of Biological Regulation,

Weizmann Institute of Science,

Rehovot 76100, Israel

e-mail: atan.gross@weizmann.ac.il
}

M. A. Andrade-Navarro

Ottawa Health Research Institute,

Ottawa, Canada

M. A. Andrade-Navarro

University of Ottawa, Faculty of Medicine, Department

of Cellular and Molecular Medicine, Ottawa and autoimmunity while accelerated apoptosis may lead to degenerative diseases. There are two major protein families which are considered as the core of the apoptotic machinery: Caspases, a family of cysteine proteases that are the major executioners of apoptosis [1], and the BCL-2 family of proteins, which are the main regulators of the process [2].

Generally, apoptosis is divided into two pathways, the extrinsic and intrinsic pathways. The extrinsic pathway is initiated by the binding of a ligand to members of the tumor necrosis factor/Fas (TNF/Fas) receptor family on the cell surface [3]. An activated receptor initiates the formation of the death-inducing signaling complex (DISC), which leads to activation of initiator caspase-8, and a downstream cascade of caspases. This ultimately leads to cleavage of cellular targets and death [4]. The intrinsic pathway involves the activation of pro-apoptotic members of the BCL-2 family that exert their function at the mitochondria by inducing the permeabilization of the outer mitochondrial membrane (OMM). This permeabilization results in the release of proapoptotic factors from the inter membrane space (IMS), such as cytochrome $c(\mathrm{Cyt} c)$, to activate effector caspases [5]. The two pathways are interconnected via the BH3-only protein $\mathrm{BID}$, which is cleaved by caspase- 8 . Truncated BID (tBID) translocates to the mitochondria and triggers the activation of the pro-apoptotic BCL-2 family members BAX and BAK, leading to OMM permeabilization. In the extrinsic pathway, this permeabilization serves as an amplification loop for the activation of effector caspases [6-8].

\section{The BCL-2 family}

The BCL-2 proto-oncogene was the first member of this family to be discovered. This gene was found at the chromosomal 
breakpoint of $\mathrm{t}(14 ; 18)$ bearing human B-cell lymphomas. Since then, many other genes homologous to BCL-2 have been found including both pro-apoptotic (e.g., BAX) and anti-apoptotic (e.g., BCL- $\mathrm{X}_{\mathrm{L}}$ ) proteins [2]. The BCL-2 family members possess up to four conserved BCL-2 homology (BH) domains designated $\mathrm{BH} 1, \mathrm{BH} 2, \mathrm{BH} 3$ and $\mathrm{BH} 4$, which correspond to $\alpha$ helical segments [9]. Most family members display sequence conservation in the first three domains (multidomain molecules). Deletion and mutagenesis studies argue that the amphipathic $\alpha$ helical BH3 domain serves as the critical death domain in the pro-apoptotic members. This concept is supported by an emerging subset of "BH3-only" pro-apoptotic members (e.g., BID) that display sequence homology only within the $\mathrm{BH} 3$ domain $[10,11]$. A major characteristic of the BCL-2 family members is their frequent ability to form homo- as well as heterodimers suggesting neutralizing competition between these proteins. A further characteristic of functional significance is their ability to associate with membranes. Many BCL-2 family members also carry a C-terminal hydrophobic domain, which is essential for their targeting to membranes such as the OMM. The molecular mechanism of OMM permeabilization, which is a key event in the mitochondrial death pathway, is poorly understood. One school of thought proposes that BCL-2 family members themselves regulate OMM permeability by virtue of their ability to form autonomous channels. This idea emerged from the structural similarity between BCL- $\mathrm{X}_{\mathrm{L}}$ and the pore forming region of bacterial toxins, and the ability of recombinant BCL- $\mathrm{X}_{\mathrm{L}}$, BCL-2, BAX and BID to form ionchannels in artificial membranes with distinct characteristics $[12,13]$. It is yet to be seen whether this ability is of physiological significance. On the other hand there is a growing body of literature suggesting that BCL-2 family members induce OMM permeabilization by regulating the function of resident mitochondrial proteins that in healthy cells function in the maintenance of cell viability, and adopt either a pro- or anti-apoptotic role, in apoptotic cells. In this review we will focus on the involvement of several mitochondrial carriers and pores in this process.

\section{The involvement of VDAC in apoptosis}

The voltage dependent anion channel (VDAC) is a highly conserved OMM protein that belongs to the porin family. This family forms aqueous channels in membranes that act as pathways for the movement of metabolites by passive diffusion. The mitochondrial channel was first discovered by Colombini $[14,15]$, who demonstrated its voltage dependency and ion selectivity, with the open state showing preference to anions, and narrowed channel size, caused by alteration in membrane potential, which shows preference to cations.
The involvement of VDAC in the process of apoptosis is unquestionable and extensively reviewed [16-18]. However the molecular mechanism of VDAC regulation in the apoptotic process is highly controversial. One theory promotes the assumption that VDAC serves as the entity that permeabilizes the OMM during apoptosis and therefore is the route through which Cyt $c$ and other IMS proteins exit the mitochondria [16]. This theory is based on the observation that several BCL-2 family members interact with VDAC [19-22]. It was reported, using proteoliposomes and electrophysiological measurements, that BCL- $\mathrm{X}_{\mathrm{L}}$ promotes the closure of VDAC whereas BAX together with VDAC form a mega channel large enough for the passage of Cyt $c$ [21]. Banerjee et al. argued that this increase in the pore size of VDAC by BAX can occur only in the presence of tBID [23]. The regulatory action of BAX on VDAC is further demonstrated by the use of a VDAC-disrupted yeast strain that shows no Cyt $c$ release upon BAX expression [20, 24]. Furthermore BAX induced Cyt $c$ release can be blocked by specific anti-VDAC antibodies [25], or by a VDAC inhibitor [26]. Interestingly, it was reported that tBID does not have an effect on the function of VDAC [27]. In this study, the authors suggested that the effect of tBID on OMM permeabilization is through another unidentified OMM protein. The importance of VDAC for OMM permeabilization is further supported by recent evidence that overexpression of VDAC induces apoptosis in various cell types, and this effect is inhibited by BCL-2 and VDAC inhibitors [28, 29].

In complete contradiction to the above, there is another theory which claims that in healthy cells VDAC is constitutively open allowing the free exchange of metabolites (e.g., ATP/ADP), whereas in apoptotic cells closure of VDAC blocks the passage of metabolites, thereby inhibiting respiration leading to mitochondrial dysfunction [17]. This theory is supported by data showing no influence of BAX on the properties of VDAC channels under a variety of conditions [30], and an inability to detect a direct interaction between BAX and VDAC in several experimental systems $[30,31]$. The holders of this theory also question the results obtained with the expression of BAX in the VDAC-disrupted yeast strain, as it appears that although release of Cyt $c$ was blocked, there was no influence on overall cell death, induced by similar BAX levels in both disrupted and wild type cells [32]. Furthermore, it was reported that removal of growth factors leads to adenine nucleotide exchange deficiency, most likely accounted by the closure of VDAC. BCL- $\mathrm{X}_{\mathrm{L}}$ restores the adenine nucleotide exchange by promoting VDAC opening [33, 34]. Along that line, there is also a report showing that tBID induces the closure of VDAC [30]. One of the outcomes of VDAC closure is the accumulation of metabolites in the IMS, which exert osmotic pressure leading to matrix swelling and subsequent OMM rupture. However, this mode of OMM permeabilization is rarely 
associated with apoptosis but rather with necrosis [35], and was also not observed in the experimental systems described above. Therefore the assumption is that release of apoptogenic factors from the IMS in this setting, must be controlled by another channel(s) [17]. Indeed, previous reports demonstrated the identification of the mitochondrial apoptosis induced channel (MAC) that has functional characteristics which are different from VDAC, and whose activity was detected in the VDAC-disrupted yeast strain [36-39].

\section{The involvement of mitochondrial carrier proteins in apoptosis}

The mitochondrial carrier (MC) protein family comprises of membrane embedded proteins that catalyze the exchange of solutes across the IMM (reviewed in [40, 41]). These carriers provide a link between mitochondria and cytosol, which is indispensable for the metabolic activities performed by mitochondria. The conserved features of this protein family are a tripartite structure made up of three tandem repeats of about 100 amino acids in length and known as the mitochondrial carrier domain (Mito_Carr domain). Each Mito_Carr domain contains two transmembrane $\alpha$-helical domains connected by an extensive hydrophilic region (see for an example, the structure of the ADP/ATP translocase in Fig. 1, left panels).

Based on the sequence information together with biochemical analysis of individual MCs it was proposed that the overall structure of each MC monomer is made up of six $\alpha$-helices transversing the IMM and connected by hydrophilic loops, with both $\mathrm{N}$ and $\mathrm{C}$ termini facing the IMS. Reconstitution into liposomes and complementation experiments in yeast revealed also common functional characteristics between the different MCs, although the substrates transported differ widely in their structure, charge and size [40].

\subsection{ADP/ATP translocase (ANT)}

The ADP/ATP translocase (or adenine nucleotide translocator; ANT) is one of the most abundant mitochondrial proteins and was the first MC to be identified, purified and sequenced [42]. Its fundamental role is to transport ATP, which is synthesized inside the mitochondria, to the cytosol, in exchange for ADP. The exchange creates an electrical gradient $\left(\mathrm{ATP}^{4-} / \mathrm{ADP}^{3-}\right)$ that drives the exchange itself [43]. Under normal settings, ANT functions as a highly specific transporter, having two conformational states. This is exhibited by the binding of its two potent inhibitors: carboxyatractyloside, which binds at the cytosolic side of the protein and fixes it in the "c"-conformation, and bongkrekic acid, which binds at the matrix side of the protein and fixes it in the " $m$ "-conformation [44]. In humans, there are three known isoforms of ANT, which differ in their expression level and tissue distribution [45-47]. Under challenging conditions, especially $\mathrm{Ca}^{2+}$ overload and oxidative stress, mitochondria undergo massive swelling and depolarization, known as the mitochondrial permeability transition (mPT) [48]. This phenomenon is the result of the opening of a non-specific large pore in the IMM, the permeability transition pore (PTP) [49]. Although the exact composition of this pore is still a debate, it is widely accepted that ANT is a core protein of this pore (also reviewed in $[50,51])$. Notably, mitochondria from livers of ANT1, ANT2 double-knockout mice were demonstrated to undergo $\mathrm{Ca}^{2+}$-induced mitochondrial swelling, although at a higher threshold $[52,53]$. This phenotype was proposed to be the result of functional compensation by other carrier proteins, based on the structural and functional resemblance between these family proteins. Additional proteins that were proposed to be part of the PTP complex (or to influence its activity) are hexokinase, creatine kinase (CK), the peripheral benzodiazepine receptor (PBR), the mitochondrial matrix cyclophilin D (Cyp D) and VDAC, which was co- purified with ANT on a GST-Cyp D affinity column [54]. However work of others argue that VDAC is not an essential component of the PTP but rather regulates its activity only under certain settings [55].

The involvement of ANT in mPT was demonstrated by the fact that different ANT ligands can affect PTP opening. Reagents that stabilize the "c"-conformation such as carboxyatractyloside, sensitize mPT to $\mathrm{Ca}^{2+}$ while reagents that stabilize the " $m$ "-conformation such as ADP and bongkrekic acid, made mPT less sensitive to $\mathrm{Ca}^{2+}$ [56-59]. Direct evidence for the presence of ANT in the PTP complex came from work of Woodfield et al. [60] that used a Cyp D affinity column to identify proteins that are part of the PTP. The only protein that retained on their column was the ANT. Furthermore, the binding of ANT to the column was blocked by pretreatment with cyclosporin A, a specific inhibitor of Cyp $D$ and a known effector of PTP opening, and enhanced when the mitochondria used were subjected to oxidative stress. In a similar experiment, Crompton et al. [54] also purified ANT using a GST-Cyp D fusion protein. The interaction between ANT and Cyp D was later demonstrated by reconstitution of recombinant proteins into lipid bilayers that showed the formation of a cyclosporine A-sensitive channel, suggesting that ANT and Cyp D are the only proteins that are essential for the formation of the pore.

\subsubsection{ANT, BAX and tBID}

BAX was shown to directly interact with ANT both by coimmunoprecipitation and by the yeast two hybrid system [61]. Moreover, this interaction was shown to be important for optimal opening of the PTP in response to atractyloside, both in isolated mitochondria and by in-vitro experiments using reconstituted ANT and BAX [62]. This effect of BAX 


\section{$\frac{\text { ADP/ATP translocase } 1}{(A N T 1)}$ (ANT1)}
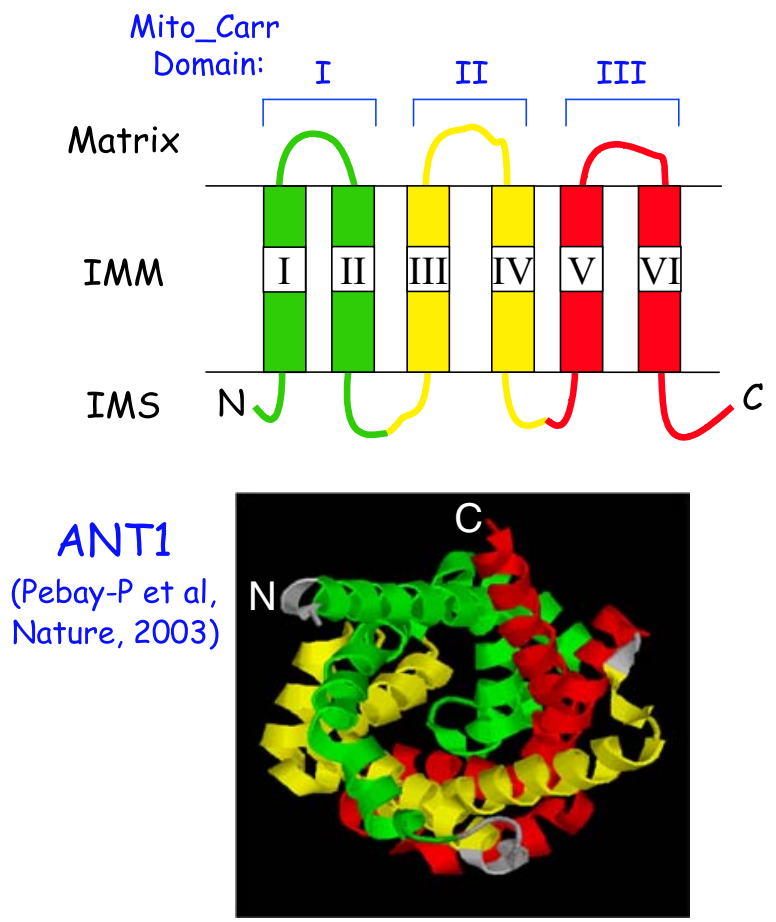

Fig. 1 Comparison of the sequences of ANT1 and hMtch2 and threading of hMtch 2 to the ANT1 structure. Top: comparison of the sequences of the bovine ADP/ATP translocase 1 [also known as adenine nucleotide translocator 1 (ANT1), or solute carrier family 25 member 4] and hMtch2. ANT1 is a 298 amino acids long sequence composed of three tandem Mito_Carr domains according to Pfam [84] here coloured in green (from amino acids 6 to 101), yellow (from 126 to 206), and red (from 206 to 297), respectively. hMtch2, with a similar length of 303 amino acids, apparently has a single middle Mito_Carr domain from positions 123 to 206 (coloured in yellow). However, sequence comparison indicates that hMtch2 and its homologous sequences such as hMtch1 or their common ancestor in the fly Mtch have extended homology into the neighbouring fragments of the $\mathrm{N}$ - and C-terminal Mito_Carr domains of ANT1 including TM helices 2 and 5 (fragments 43-122 and 207-248 in hMtch2, coloured in green and red, respectively). Iterative sequence searches using PSIBLAST [85] with the unmatched $\mathrm{N}$ - and C-terminal fragments of hMtch2 (1-42 and 249-303) did not detect any sequences outside the Mtch family. However, we observed that a chimeric sequence constructed using the 249-303 followed by

was inhibited by BCL-2 which was also shown to directly interact with ANT and compete with BAX [62].

Zamzami et al. [63] demonstrated that tBID-induced permeabilization of the OMM is inhibited by both cyclosporine $\mathrm{A}$ and bongkrekic acid, and therefore suggested a functional interaction between tBID and ANT. Since there is no evidence for a direct physical interaction between tBID and ANT, it is tempting to speculate that tBID affects ANT function by interacting with lipids surrounding it. ANT is tightly bound to six molecules of cardiolipin. These interactions are essential for the normal function of ANT [64-66]. In the last

\section{Mitochondrial carrier homolog 2 (M+ch2)}
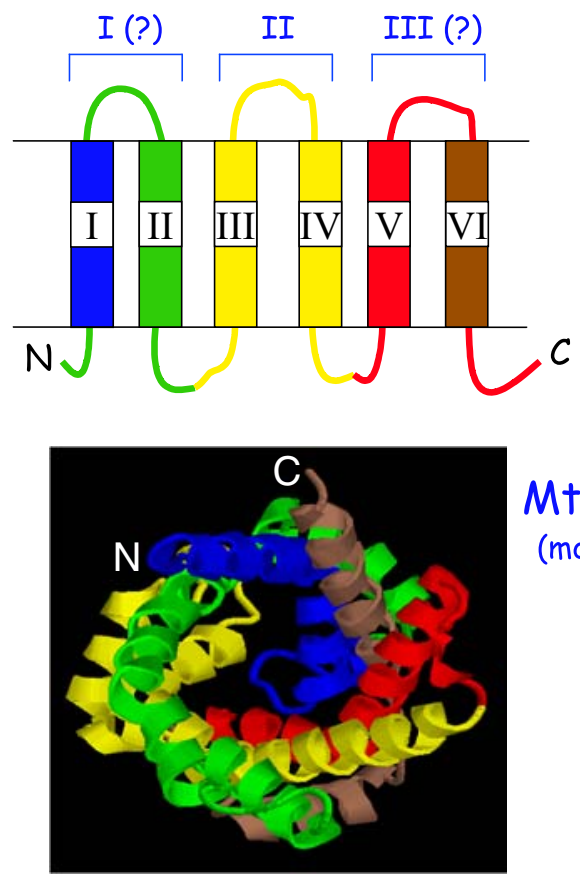

M+ch2

(model)

the 1-42 was matched to the complete Pfam Mito_Carr profile with an E-value of 0.081 . This suggests that: (1) the actual evolutionary unit in ANT1 would not be the currently defined Mito_Carr domains comprising TM1/TM2, TM3/TM4. and TM5/TM6, but TM2/TM3, TM4/TM5, and TM6/TM1 (the formation of a hybrid N- terminal Cterminal domain has also been described for beta barrels of repeatslike in Kelch-as is known as a "clasp" mechanism that helps the stability of the closing structure); (2) in the Mtch family, the domains TM2/TM3 and TM4/TM5 are present, but the hybrid clasp domain is a very divergent reversed TM1/TM6. Bottom: To further support this hypothesis, we threaded the sequence of hMtch2 into the structure of ANT1 (PDB:1 lock) [79] using the Phyre web tool [Kelley, unpublished; http://www.imperial.ac.uk/phyre/]. The fold recognition of ANT1 by hMtch2 received an E-value of 2e-15 for a length of 298 amino acids on ANT1 (complete sequence). For illustration of the spatial position of the units the structure of ANT1 (left) and the resulting modeled structure for hMtch2 (right) are presented. The orientation is such that the axis of symmetry of the barrel runs perpendicular to the page with the TMs running almost perpendicular to the page

several years it has been established that tBID also intimately interacts with cardiolipin $[67,68]$, and that this interaction is involved in regulating the mitochondrial death pathway [69-73]. Thus, the tBID-cardiolipin interaction might drive a conformational change in ANT, which might trigger the binding of BAX.

\subsection{Mitochondrial carrier homolog 2 (Mtch2)}

Previously we reported that in $\mathrm{TNF} \alpha$-activated hematopoietic FL5.12 cells, p15 tBID forms a $\sim 45 \mathrm{kD}$ cross-linkable 
complex at the mitochondria [74]. More recently, we demonstrated that this complex represents an interaction between tBID and mitochondrial carrier homolog 2 (Mtch2), a novel and previously uncharacterized $33 \mathrm{kD}$ protein $[75,76]$. Mtch2 was first identified as a putative ORF obtained from CD34+ hematopoetic stem/progenitor cells [77], named after its single conserved Mito_Carr domain (Mito_Carr domain II; see Fig. 1, right panel). Mtch2 (also known as Mimp) was also shown to be induced by Met-hepatocyte growth factor/scatter factor signaling [78].

Mtch2 has several close relatives, which together form a "sister family" separate from the other mitochondrial carrier proteins $[75,76]$. All Mtch family members contain a single conserved Mito_Carr domain, and can be aligned across their entire length. Having only one conserved Mito_Carr domain, it was first predicted to have three trans-membrane (TM) domains (two that form the Mito_Carr domain, and a third C-terminal one) $[75,76]$. However, sequence similarity analysis and threading using the 3D structure of ANT1 [79] suggests that the overall structure of Mtch2 resembles the typical structure of MCs (six TM domains connected with hydrophilic loops; Fig. 1, right panels). Of special note, Mtch2 does carry unique features [the $\mathrm{N}$-terminal helix of Mito_Carr domain I (blue) and the C-terminal helix of Mito_Carr domain III (brown)], which are different from the helices in ANT (compare bottom panels in Fig. 1), suggesting that Mtch2 (and probably its close relatives) are related to MCs but distinct.

Biochemical analysis of Mtch2 reveals that it is exposed on the surface of mitochondria, with all its TM domains likely transversing the OMM ([76], and data not shown). This feature seems to be unique to Mtch2, as all other MCs are IMM proteins. This data also fits well with the fact that tBID, which does not seem to reach the IMM [80], directly interacts with Mtch2 [76]. The unique localization of Mtch2 may be explained by its amino acid sequence. As noted earlier, the amino acid sequence of the Mtch2 protein contains a central conserved region (including a region corresponding to Mito_Carr domain II in other MCs), while the N- and $\mathrm{C}$-terminal fragments that are nearby in space and form probably a domain (see Fig. 1, right panels) are less conserved. Studies using deletion mutants of ANT have demonstrated that the insertion mechanism of ANT to the IMM requires cooperate binding of all three Mito_Carr domains to components of the import complexes of both the outer and inner membranes (TOMs and TIMs, respectively; [81]). However the exact residues that are involved in the binding are unknown. Thus, it is possible that the conserved Mito_Carr domain II allows the binding of Mtch2 to the TOM complex, but not its transfer to the TIM complex in the IMM, and therefore it remains stuck in the OMM.

Blue-native gel electrophoresis analysis performed on mitochondria of both healthy cells and cells signaled to die by
$\mathrm{TNF} \alpha$ revealed that Mtch2 resides in an approximately 185 $\mathrm{kD}$ resident complex, and activation with TNF $\alpha$ leads to the recruitment of both tBID and BAX to this complex [76]. The recruitment of pro-apoptotic BCL-2 family members to the complex seems to be important for the induction of the mitochondrial death pathway, since over expression of BCL- $\mathrm{X}_{\mathrm{L}}$, which inhibits TNF $\alpha$-induced Cyt $c$ release, also inhibits the recruitment of both tBID and BAX to the Mtch2-resident complex [76].

\subsubsection{What might be the function of Mtch2?}

The function of Mtch2 is still unknown. It was previously reported that over expression of human presenilin-1-associated protein (PSAP, also known as human Mtch1, a protein with 48\% identity to human Mtch2 [76]), induces Cyt $c$ release, caspase activation and apoptosis, implying a pro-apoptotic role for this protein [82]. Over expression of Mtch2 in 293T and HeLa cells, however, did not induce apoptosis [76]. It is important to note that the lack of an effect could be due to the low expression levels that were obtained. Interestingly, it was reported that overexpression of Mtch2/Mimp in mammary adenocarcinoma DA3 cells did not induce apoptosis, but did cause loss of mitochondrial membrane potential [78, 83]. These studies proposed a role for Mtch 2 in regulation of cellular growth, motility and tumorigenicity.

Since the members of the Mtch family are close relatives of MC proteins, they might function as carrier proteins, transporting certain metabolites across the OMM rather than across the IMM. This is supported by their apparently similar arrangement of transmembrane domains (Fig. 1). However, the different terminals make them distinct and less symmetrical than their MC partners. The divergent hybrid N-terminal C-terminal domain might hold protein interaction sites specific to the Mtch family.

The fact that both tBID and BAX co-reside with the Mtch2 resident complex in apoptotic cells suggests that Mtch2 might be involved in tBID-induced BAX activation/oligomerization. It is known that $\mathrm{BIID}$ activates BAX via its $\mathrm{BH} 3$ domain, however the interaction of tBID with Mtch2 is BH3- and BAX/BAK-independent [74]. This may imply that Mtch2 serves as an anchor protein for tBID, that stably anchors and/or correctly positions tBID in the membrane, to allow the activation of BAX via its exposed $\mathrm{BH} 3$ domain.

\section{Concluding remark}

Mitochondria are Jekyll and Hyde entities. In healthy cells they are the power plants providing energy to sustain life activities. At the same time mitochondria also serve as the storage compartment for apoptogenic factors which are 
released upon death stimuli. This dual function on the level of the organelle is maintained also on the level of individual mitochondrial proteins. As discussed in this review, mitochondrial carriers and pores, which in healthy cells function to maintain cell viability, might alter their function under certain stress conditions to promote the mitochondrial apoptotic program. We believe that the list of mitochondrial carriers and pores that are involved in this "balancing act" between cell life and death is likely to increase in the future. In a way one can look at this set of proteins as the link between the major regulators of apoptosis (BCL-2 proteins) and the major executioners (caspases), and therefore are likely to represent excellent therapeutic targets.

Acknowledgments Research in my laboratory is supported in part by the Israel Science Foundation, the Israel Cancer Association (through a donation from Teva Pharmaceutical Industries Ltd.), the A-T Children's Project, Minerva Stiftung, MDM ICR Research Award, the USA-Israel Binational Science Foundation (BSF), Joint German-Israeli Research Program in Cancer Research (IMOS-DKFZ), the Willner Family Center for Vascular Biology, and Mr. and Mrs. Stanley Chais. A.G. is the incumbent of the Armour Family Career Development Chair of Cancer Research.

\section{References}

1. Shi Y (2002) Mechanisms of caspase activation and inhibition during apoptosis. Mol Cell 9:459-470

2. Danial NN, Korsmeyer SJ (2004) Cell death: critical control points. Cell 116:205-219

3. Varfolomeev EE, Ashkenazi A (2004) Tumor necrosis factor: an apoptosis JuNKie? Cell 116:491-497

4. Medema JP, Scaffidi C, Kischkel FC et al (1997) FLICE is activated by association with the CD95 death-inducing signaling complex (DISC). Embo J 16:2794-2804

5. Wang $X$ (2001) The expanding role of mitochondria in apoptosis. Genes Dev 15:2922-2933

6. Luo X, Budihardjo I, Zou H, Slaughter C, Wang X (1998) Bid, a $\mathrm{Bcl} 2$ interacting protein, mediates cytochrome $c$ release from mitochondria in response to activation of cell surface death receptors. Cell 94:481-490

7. Li H, Zhu H, Xu CJ, Yuan J (1998) Cleavage of BID by caspase 8 mediates the mitochondrial damage in the Fas pathway of apoptosis. Cell 94:491-501

8. Gross A, Yin XM, Wang K et al (1999) Caspase cleaved BID targets mitochondria and is required for cytochrome $c$ release, while BCL-XL prevents this release but not tumor necrosis factor-R1/Fas death. J Biol Chem 274:1156-1163

9. Cory S, Adams JM (2002) The Bcl2 family: regulators of the cellular life-or-death switch. Nat Rev Cancer 2:647-656

10. Willis SN, Adams JM (2005) Life in the balance: how BH3only proteins induce apoptosis. Curr Opin Cell Biol 17:617625

11. Huang DC, Strasser A (2000) BH3-Only proteins-essential initiators of apoptotic cell death. Cell 103:839-842

12. Schendel SL, Montal M, Reed JC (1998) Bcl-2 family proteins as ion-channels. Cell Death Differ 5:372-380

13. Muchmore SW, Sattler M, Liang H et al (1996) X-ray and NMR structure of human Bcl-xL, an inhibitor of programmed cell death. Nature 381:335-341
14. Colombini M (1979) A candidate for the permeability pathway of the outer mitochondrial membrane. Nature 279:643-645

15. Colombini M (1980) Structure and mode of action of a voltage dependent anion-selective channel (VDAC) located in the outer mitochondrial membrane. Ann NY Acad Sci 341:552-563

16. Tsujimoto Y, Shimizu S (2002) The voltage-dependent anion channel: an essential player in apoptosis. Biochimie 84:187-193

17. Rostovtseva TK, Tan W, Colombini M (2005) On the role of VDAC in apoptosis: fact and fiction. J Bioenerg Biomembr 37:129-142

18. Bernardi P, Petronilli V, Di Lisa F, Forte M (2001) A mitochondrial perspective on cell death. Trends Biochem Sci 26:112-117

19. Shimizu S, Konishi A, Kodama T, Tsujimoto Y (2000) BH4 domain of antiapoptotic Bcl-2 family members closes voltagedependent anion channel and inhibits apoptotic mitochondrial changes and cell death. Proc Natl Acad Sci USA 97:3100-3105

20. Shimizu S, Narita M, Tsujimoto Y (1999) Bcl-2 family proteins regulate the release of apoptogenic cytochrome $c$ by the mitochondrial channel VDAC. Nature 399:483-487

21. Shimizu S, Ide T, Yanagida T, Tsujimoto Y (2000) Electrophysiological study of a novel large pore formed by Bax and the voltage-dependent anion channel that is permeable to cytochrome c. J Biol Chem 275:12321-12325

22. Shi Y, Chen J, Weng C et al (2003) Identification of the proteinprotein contact site and interaction mode of human VDAC1 with Bcl-2 family proteins. Biochem Biophys Res Commun 305:989-996

23. Banerjee J, Ghosh S (2004) Bax increases the pore size of rat brain mitochondrial voltage-dependent anion channel in the presence of tBid. Biochem Biophys Res Commun 323:310-314

24. Shimizu S, Shinohara Y, Tsujimoto Y (2000) Bax and Bcl-xL independently regulate apoptotic changes of yeast mitochondria that require VDAC but not adenine nucleotide translocator. Oncogene 19:4309-4318

25. Shimizu S, Matsuoka Y, Shinohara Y, Yoneda Y, Tsujimoto Y (2001) Essential role of voltage-dependent anion channel in various forms of apoptosis in mammalian cells. J Cell Biol 152:237-250

26. Ferri KF, Jacotot E, Blanco J et al (2000) Apoptosis control in syncytia induced by the HIV type 1-envelope glycoprotein complex: role of mitochondria and caspases. J Exp Med 192:1081-1092

27. Shimizu S, Tsujimoto Y (2000) Proapoptotic BH3-only Bcl-2 family members induce cytochrome $c$ release, but not mitochondrial membrane potential loss, and do not directly modulate voltage-dependent anion channel activity. Proc Natl Acad Sci USA 97:577-582

28. Zaid H, Abu-Hamad S, Israelson A, Nathan I, Shoshan-Barmatz V (2005) The voltage-dependent anion channel-1 modulates apoptotic cell death. Cell Death Differ 12:751-760

29. Shoshan-Barmatz V, Israelson A, Brdiczka D, Sheu SS (2006) The voltage-dependent anion channel (VDAC): function in intracellular signalling, cell life and cell death. Curr Pharm Des 12:2249-2270

30. Rostovtseva TK, Antonsson B, Suzuki M, Youle RJ, Colombini M, Bezrukov SM (2004) Bid, but not Bax, regulates VDAC channels. J Biol Chem 279:13575-13583

31. Mikhailov V, Mikhailova M, Pulkrabek DJ, Dong Z, Venkatachalam MA, Saikumar P (2001) Bcl-2 prevents Bax oligomerization in the mitochondrial outer membrane. J Biol Chem 276:1836118374

32. Polcic P, Forte M (2003) Response of yeast to the regulated expression of proteins in the Bcl-2 family. Biochem J 374:393-402

33. Vander Heiden MG, Chandel NS, Li XX, Schumacker PT, Colombini M, Thompson CB (2000) Outer mitochondrial membrane permeability can regulate coupled respiration and cell survival. Proc Natl Acad Sci USA 97:4666-4671

34. Vander Heiden MG, Li XX, Gottleib E, Hill RB, Thompson CB, Colombini M (2001) Bcl-xL promotes the open configuration of the voltage-dependent anion channel and metabolite 
passage through the outer mitochondrial membrane. J Biol Chem 276:19414-19419

35. Tsujimoto Y, Shimizu S (2000) Bcl-2 family: life-or-death switch. FEBS Lett 466:6-10

36. Pavlov EV, Priault M, Pietkiewicz D et al (2001) A novel, high conductance channel of mitochondria linked to apoptosis in mammalian cells and Bax expression in yeast. J Cell Biol 155:725-731

37. Martinez-Caballero S, Dejean LM, Jonas EA, Kinnally KW (2005) The role of the mitochondrial apoptosis induced channel MAC in cytochrome $c$ release. J Bioenerg Biomembr 37:155-164

38. Dejean LM, Martinez-Caballero S, Kinnally KW (2006) Is MAC the knife that cuts cytochrome $c$ from mitochondria during apoptosis? Cell Death Differ 13:1387-1395

39. Dejean LM, Martinez-Caballero S, Guo L et al (2005) Oligomeric Bax is a component of the putative cytochrome $c$ release channel MAC, mitochondrial apoptosis-induced channel. Mol Biol Cell $16: 2424-2432$

40. Palmieri F (2004) The mitochondrial transporter family (SLC25): physiological and pathological implications. Pflugers Arch 447:689-709

41. Arco AD, Satrustegui J (2005) New mitochondrial carriers: an overview. Cell Mol Life Sci 62:2204-2227

42. Aquila H, Misra D, Eulitz M, Klingenberg M (1982) Complete amino acid sequence of the ADP/ATP carrier from beef heart mitochondria. Hoppe Seylers Z Physiol Chem 363:345-349

43. Klingenberg M (1980) The ADP-ATP translocation in mitochondria, a membrane potential controlled transport. J Membr Biol 56:97-105

44. Fiore C, Trezeguet V, Le Saux A et al (1998) The mitochondrial ADP/ATP carrier: structural, physiological and pathological aspects. Biochimie 80:137-150

45. Stepien G, Torroni A, Chung AB, Hodge JA, Wallace DC (1992) Differential expression of adenine nucleotide translocator isoforms in mammalian tissues and during muscle cell differentiation. $\mathrm{J}$ Biol Chem 267:14592-14597

46. Lunardi J, Hurko O, Engel WK, Attardi G (1992) The multiple ADP/ATP translocase genes are differentially expressed during human muscle development. J Biol Chem 267:15267-15270

47. Barath P, Luciakova K, Hodny Z, Li R, Nelson BD (1999) The growth-dependent expression of the adenine nucleotide translocase-2 (ANT2) gene is regulated at the level of transcription and is a marker of cell proliferation. Exp Cell Res 248:583-588

48. Hunter DR, Haworth RA, Southard JH (1976) Relationship between configuration, function, and permeability in calcium-treated mitochondria. J Biol Chem 251:5069-5077

49. Petronilli V, Szabo I, Zoratti M (1989) The inner mitochondrial membrane contains ion-conducting channels similar to those found in bacteria. FEBS Lett 259:137-143

50. Halestrap AP, McStay GP, Clarke SJ (2002) The permeability transition pore complex: another view. Biochimie 84:153-166

51. Brenner C, Grimm S (2006) The permeability transition pore complex in cancer cell death. Oncogene 25:4744-4756

52. Kokoszka JE, Waymire KG, Levy SE et al (2004) The ADP/ATP translocator is not essential for the mitochondrial permeability transition pore. Nature 427:461-465

53. Halestrap AP (2004) Mitochondrial permeability: dual role for the ADP/ATP translocator? Nature 427:461-465

54. Crompton M, Virji S, Ward JM (1998) Cyclophilin-D binds strongly to complexes of the voltage-dependent anion channel and the adenine nucleotide translocase to form the permeability transition pore. Eur J Biochem 258:729-735

55. Halestrap AP, Doran E, Gillespie JP, O’Toole A (2000) Mitochondria and cell death. Biochem Soc Trans 28:170-177

56. Le Quoc K, Le Quoc D (1988) Involvement of the ADP/ATP carrier in calcium-induced perturbations of the mitochondrial inner membrane permeability: importance of the orientation of the nucleotide binding site. Arch Biochem Biophys 265:249-257

57. Hunter DR, Haworth RA (1979) The Ca2+-induced membrane transition in mitochondria. I. The protective mechanisms. Arch Biochem Biophys 195:453-459

58. Halestrap AP, Davidson AM (1990) Inhibition of Ca2(+)-induced large-amplitude swelling of liver and heart mitochondria by cyclosporin is probably caused by the inhibitor binding to mitochondrial-matrix peptidyl-prolyl cis-trans isomerase and preventing it interacting with the adenine nucleotide translocase. Biochem J 268:153-160

59. Halestrap AP, Woodfield KY, Connern CP (1997) Oxidative stress, thiol reagents, and membrane potential modulate the mitochondrial permeability transition by affecting nucleotide binding to the adenine nucleotide translocase. J Biol Chem 272:3346-3354

60. Woodfield K, Ruck A, Brdiczka D, Halestrap AP (1998) Direct demonstration of a specific interaction between cyclophilin-D and the adenine nucleotide translocase confirms their role in the mitochondrial permeability transition. Biochem J 336 (Pt 2):287-290

61. Marzo I, Brenner C, Zamzami N et al (1998) Bax and adenine nucleotide translocator cooperate in the mitochondrial control of apoptosis. Science 281:2027-2031

62. Marzo I, Brenner C, Kroemer G (1998) The central role of the mitochondrial megachannel in apoptosis: evidence obtained with intact cells, isolated mitochondria, and purified protein complexes. Biomed Pharmacother 52:248-251

63. Zamzami N, El Hamel C, Maisse C et al (2000) Bid acts on the permeability transition pore complex to induce apoptosis. Oncogene 19:6342-6350

64. Hoffmann B, Stockl A, Schlame M, Beyer K, Klingenberg M (1994) The reconstituted ADP/ATP carrier activity has an absolute requirement for cardiolipin as shown in cysteine mutants. J Biol Chem 269:1940-1944

65. Beyer K, Klingenberg M (1985) ADP/ATP carrier protein from beef heart mitochondria has high amounts of tightly bound cardiolipin, as revealed by $31 \mathrm{P}$ nuclear magnetic resonance. Biochemistry 24:3821-3826

66. Beyer K, Nuscher B (1996) Specific cardiolipin binding interferes with labeling of sulfhydryl residues in the adenosine diphosphate/adenosine triphosphate carrier protein from beef heart mitochondria. Biochemistry 35:15784-15790

67. Lutter M, Fang M, Luo X, Nishijima M, Xie X, Wang X (2000) Cardiolipin provides specificity for targeting of tBid to mitochondria. Nat Cell Biol 2:754-761

68. Liu J, Durrant D, Yang HS et al (2005) The interaction between tBid and cardiolipin or monolysocardiolipin. Biochem Biophys Res Commun 330:865-870

69. Liu J, Weiss A, Durrant D, Chi NW, Lee RM (2004) The cardiolipin-binding domain of Bid affects mitochondrial respiration and enhances cytochrome $c$ release. Apoptosis 9:533-541

70. Kuwana T, Mackey MR, Perkins G et al (2002) Bid, Bax, and lipids cooperate to form supramolecular openings in the outer mitochondrial membrane. Cell 111:331-342

71. Kim TH, Zhao Y, Ding WX et al (2004) Bid-cardiolipin interaction at mitochondrial contact site contributes to mitochondrial cristae reorganization and cytochrome $\mathrm{C}$ release. Mol Biol Cell 15:3061-3072

72. Gonzalvez F, Bessoule JJ, Rocchiccioli F, Manon S, Petit PX (2005) Role of cardiolipin on tBid and tBid/Bax synergistic effects on yeast mitochondria. Cell Death Differ 12:659-667

73. Gonzalvez F, Pariselli F, Dupaigne P et al (2005) tBid interaction with cardiolipin primarily orchestrates mitochondrial dysfunctions and subsequently activates Bax and Bak. Cell Death Differ $12: 614-626$

74. Grinberg M, Sarig R, Zaltsman Y et al (2002) tBID Homooligomerizes in the mitochondrial membrane to induce apoptosis. J Biol Chem 277:12237-12245 
75. Gross A (2005) Mitochondrial carrier homolog 2: a clue to cracking the BCL-2 family riddle? J Bioenerg Biomembr 37:113-119

76. Grinberg M, Schwarz M, Zaltsman Y et al (2005) Mitochondrial carrier homolog 2 is a target of tBID in cells signaled to die by tumor necrosis factor alpha. Mol Cell Biol 25:4579-4590

77. Zhang QH, Ye M, Wu XY et al (2000) Cloning and functional analysis of cDNAs with open reading frames for 300 previously undefined genes expressed in CD34+ hematopoietic stem/progenitor cells. Genome Res 10:1546-1560

78. Yerushalmi GM, Leibowitz-Amit R, Shaharabany M, Tsarfaty I (2002) Met-HGF/SF signal transduction induces mimp, a novel mitochondrial carrier homologue, which leads to mitochondrial depolarization. Neoplasia 4:510-522

79. Pebay-Peyroula E, Dahout-Gonzalez C, Kahn R, Trezeguet V, Lauquin GJ, Brandolin G (2003) Structure of mitochondrial ADP/ATP carrier in complex with carboxyatractyloside. Nature 426:39-44

80. Oh KJ, Barbuto S, Meyer N, Kim RS, Collier RJ, Korsmeyer SJ (2005) Conformational changes in BID, a pro-apoptotic BCL-2 family member, upon membrane binding. A site-directed spin labeling study. J Biol Chem 280:753- 767

81. Wiedemann N, Pfanner N, Ryan MT (2001) The three modules of ADP/ATP carrier cooperate in receptor recruitment and translocation into mitochondria. Embo J 20:951960

82. Xu X, Shi YC, Gao W et al (2002) The novel presenilin-1associated protein is a proapoptotic mitochondrial protein. J Biol Chem 277:48913-48922

83. Leibowitz-Amit R, Tsarfaty G, Abargil Y, Yerushalmi GM, Horev J, Tsarfaty I (2006) Mimp, a Mitochondrial Carrier Homologue, Inhibits Met-HGF/SF-Induced Scattering and Tumorigenicity by Altering Met-HGF/SF Signaling Pathways. Cancer Res 66:8687-8697

84. Finn RD, Mistry J, Schuster-Bockler B et al (2006) Pfam: clans, web tools and services. Nucleic Acids Res 34:D247-251

85. Altchuler SF, Madden TL, Schaffer AA et al (1997) Gapped BLAST and PSI-BLAST: a new generation of protein database search programs. Nucleic Acids Res 25:3389-3402 